$\mathbb{T}$ periodica polytechnica

Electrical Engineering and Computer Science

57/1 (2013) 3 7

doi: 10.3311/PPee.2066

http://periodicapolytechnica.org/ee

Creative Commons Attribution (1)

RESEARCH ARTICLE

\section{Prototype MEMS Capacitive Pressure Sensor Design and Manufacturing}

\author{
Tamás Kárpáti / Andrea Edit Pap / Sándor Kulinyi
}

Received 2013-03-12, revised 2013-05-28, accepted 2013-05-28

\begin{abstract}
This paper is intended to describe the design and manufacturing aspects of a simple micromachined capacitive pressure sensor working in the pressure range of 0-1000 mbar. $500 \mu \mathrm{m}$ thick Borofloat $^{\circledR} 33$ glass and silicon wafers were used as substrates. The basic transducer structure consists of a rectangular silicon membrane as deformable electrode and a fix aluminum electrode formed on the glass surface. In order to determine the exact geometry of the silicon electrode structure numerical models and simulations were applied. The thin silicon membrane was fabricated by Si bulk micromachining, i.e. anisotropic alkaline etching with electrochemical etch-stop. The two wafers were bonded together at low temperature by anodic bonding. After bonding and dicing the wafers the pressure sensors were characterized mechanically and functionally also. Our results demonstrate the functional behavior of the manufactured sensor structures and provide excellent verification of the preliminary expectations based on theoretical calculations and electromechanical simulations.
\end{abstract}

\section{Keywords}

capacitive pressure sensor $\cdot$ micromachining $\cdot M E M S \cdot$ anodic bonding

\section{Acknowledgement}

Support of the Hungarian National Research Projects KMR_12_1_2012_0107 and KMR_12_1_2012_0031 is gratefully acknowledged.

\section{Tamás Kárpáti}

Institute for Technical Physics and Materials Science, RCNS, HAS, H-1525 Budapest, P.O. Box 49., Hungary

e-mail: karpati.tamas@ttk.mta.hu

\section{Andrea Edit Pap}

Institute for Technical Physics and Materials Science, RCNS, HAS, H-1525 Budapest, P.O. Box 49., Hungary

e-mail: pap.andrea@ttk.mta.hu

\section{Sándor Kulinyi}

Weszta-T Kft., Ciklámen street 43., H-2011 Budakalász, Hungary

e-mail: automatik@weszta-t.hu

\section{Introduction}

Pressure sensors are frequently used in various applications from simple household equipment to complex process monitoring and control systems in industrial automation, vehicle industry, and medical devices. [1] Pressure sensors are capable of measuring pressure directly, and indirectly measure other parameters, e.g. fluid level, gas flow rate, speed or altitude. The working principle of these pressure sensors and transducers usually utilizes the precise measurement of a membrane / diaphragm deformation transforming the force affected on the sensing area to an electrical signal applying capacitive, piezoresistive, optical or resonance conversion method. Historically, macroscopic size sensors with metal membranes were used for detection and the evolution of the silicon micromachining opened a new way for more sensitive and miniaturized pressure sensors. [2] Nowadays surface micromachined MEMS pressure sensors integrated with CMOS circuits are investigated. [3] Depending on the sensor construction three different types of sensing methods are available. First is the absolute pressure sensor which measures the pressure relative to perfect vacuum. Second one is the gauge type: it measures the differences to the atmospheric pressure. Finally, the differential pressure sensor: it measures the difference between two pressures, one connected to each side of the sensor.

According to the application field (industrial pressure control) the proposed sensor structures were designed for working in the low pressure ranges between 0-1000 mbar.

The capacitive measurement principle was applied as read-out mechanism due to its higher expected sensitivity compared to a piezoresistive detection method. Moreover, significantly lower power consumption and temperature dependence / effect on the sensor response could be expected. However, the small sensing capacitance, the high output impedance, and the nonlinear characteristics of the sensor response are disadvantages. [4]

\section{Experimental details}

The pressure sensor structure is constructed from two independent parts. One is the single crystalline Si membrane forming a flexible electrode and the other is a Borofloat ${ }^{\circledR} 33$ glass die 
with aluminium capacitor plate (fixed electrode) and bonding pads (as shown in Fig. 1).

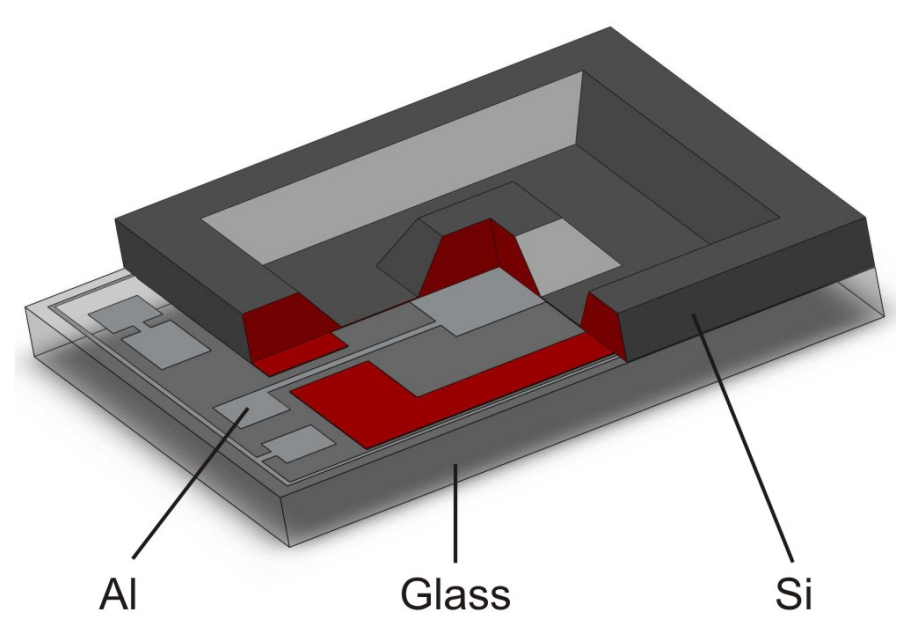

Fig. 1. Schematic drawing of the silicon-based capacitive pressure sensors.

The silicon membrane was formed by anisotropic alkaline etching, using the adequate masking to preserve the original wafer thickness at the outer frame around the membrane and at the center. The center support can improve the mechanical stability and the good tractability of the thin membrane during the processing steps. Moreover, the center mass reinforces the membrane providing for parallel capacitor electrodes even during deformation of the membrane. In order to avoid electrical shortcuts between the moving and the fix electrodes the metal contact addressing the lower electrode on the glass is located in a trench formed by smooth silicon etch.

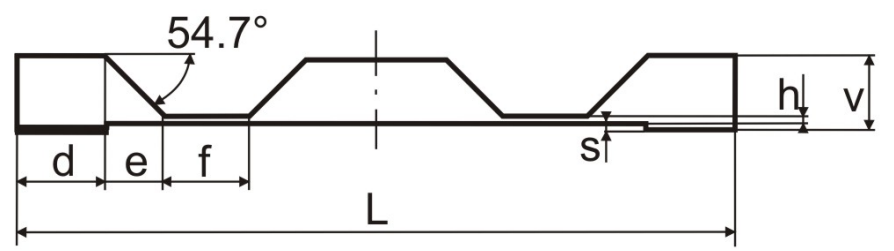

Fig. 2. The cross-sectional drawing and signs of the Si electrodes.

The general parameters of the presented device are listed in Table 1. and shown in Fig 2, [5] The zero-pressure capacitance of the device is planned to lie between 1-10 pF.

\section{Preliminary investigations by simulation}

The final functional parameters of the capacitive pressure sensors strongly depend on its geometrical dimensions. The device sensitivity shows inverse correlation to the cube of the diaphragm thickness and is proportional to the square of the relevant area of the membrane. In order to achieve optimal geometry parameters finite element modeling was applied to simulate the functional behavior of the device. The membrane area, thickness, and the size of the center mass were varied in the applied model. Based on the simulation results the most robust and sen- sitive structure in the range of 0-1000 mbar (see Table 1) was selected and manufactured. Fig. 3 shows a representative displacement distribution of the $20 \mu \mathrm{m}$ thick membrane under 1.25 bar pressure. [5]

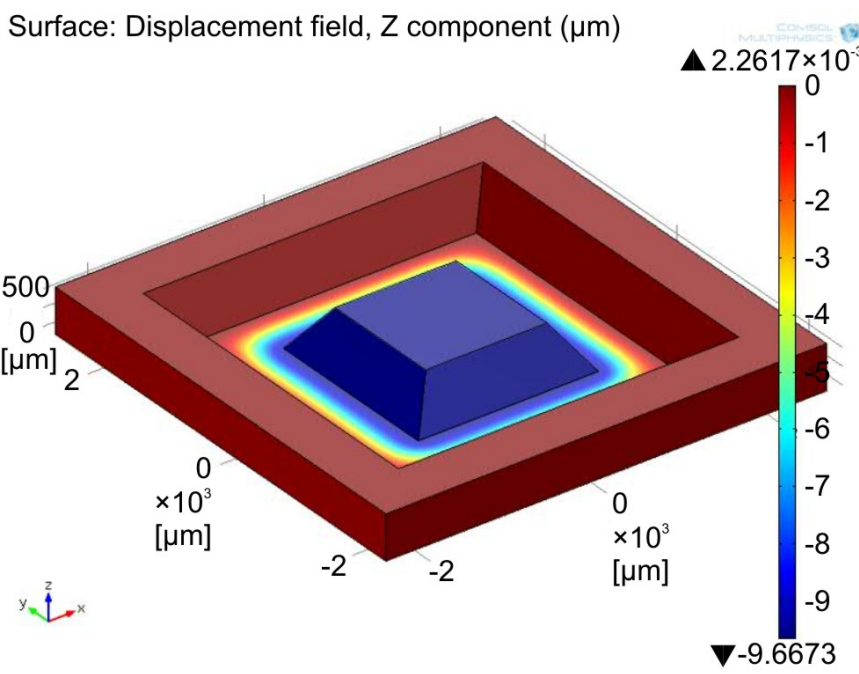

Fig. 3. Representation of simulated $20 \mu \mathrm{m}$ thick membrane deformation, applying 1.25 bar pressure.

Tab. 1. The general parameters of the device.

\begin{tabular}{lc}
\hline Si chip & $\begin{array}{c}5 \times 5 \times 0.5 \mathrm{~mm}(\mathrm{~L} \times \mathrm{L} \times \mathrm{v}) \\
d=500 \mu \mathrm{m} \quad e=340 \mu \mathrm{m} \\
f=1000 \mu \mathrm{m} \quad s=10 \mu \mathrm{m} \\
h=20 \mu \mathrm{m}\end{array}$ \\
\hline Glass electrode & $\begin{array}{c}6 \times 5 \times 0.5 \mathrm{~mm} \\
\text { with } 1 \mu \mathrm{m} \text { thick Al layer }\end{array}$ \\
\hline
\end{tabular}

\section{Membrane preparation process}

The device development was focused on the reliable, reproducible membrane fabrication, especially on the uniform mem-

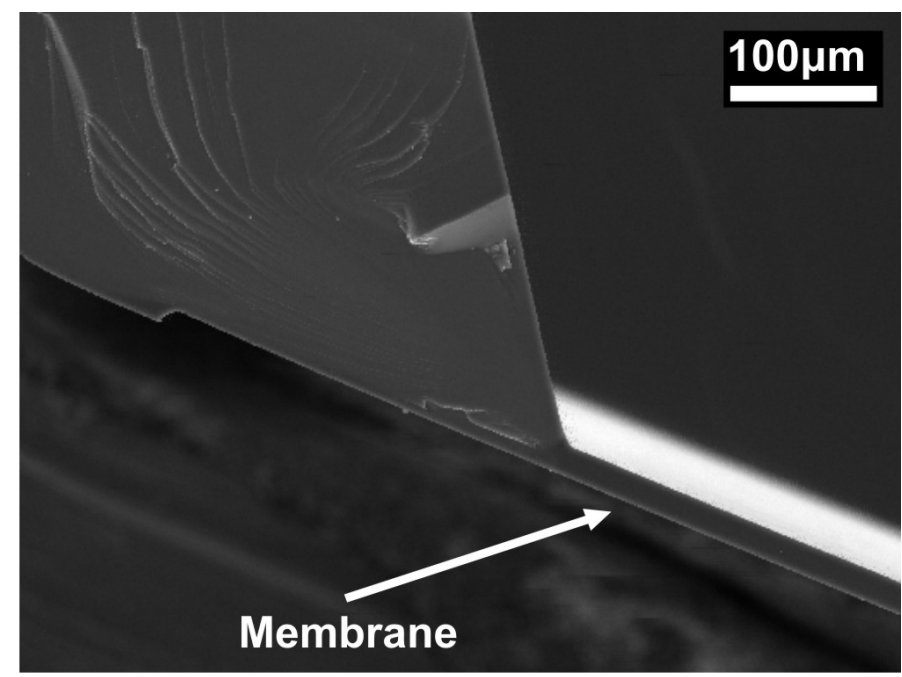

Fig. 4. Membrane formed by electrochemical etch-stop method (from the top side) and isotropic etching (from the bottom side). 
brane thickness, homogeneity and smooth surface. The membrane formation was achieved by Si bulk micromachining technology utilizing anisotropic alkaline etching combined with Electrochemical Etch-Stop (ECES) to accurately control the final membrane thickness. [6] The ECES method is applicable to significantly decrease the vertical etching rate at a $p-n$ junction developed by adequate doping processes by electrochemical formation of a Silicon-Oxide passivation layer on the silicon surface. The homogeneity of the formed silicon membrane is clearly demonstrated by Fig. 4.

The $10 \mu \mathrm{m}$ deep cavity forming the gap of the capacitor between the silicon and metal electrode was developed by isotropic silicon etching. From a technological aspect the most critical parameter here is the surface roughness, since residual inhomogeneities on the membrane surface restrict the upper detection limit of the device, and/or the mechanical durability and performance of the moving electrode.

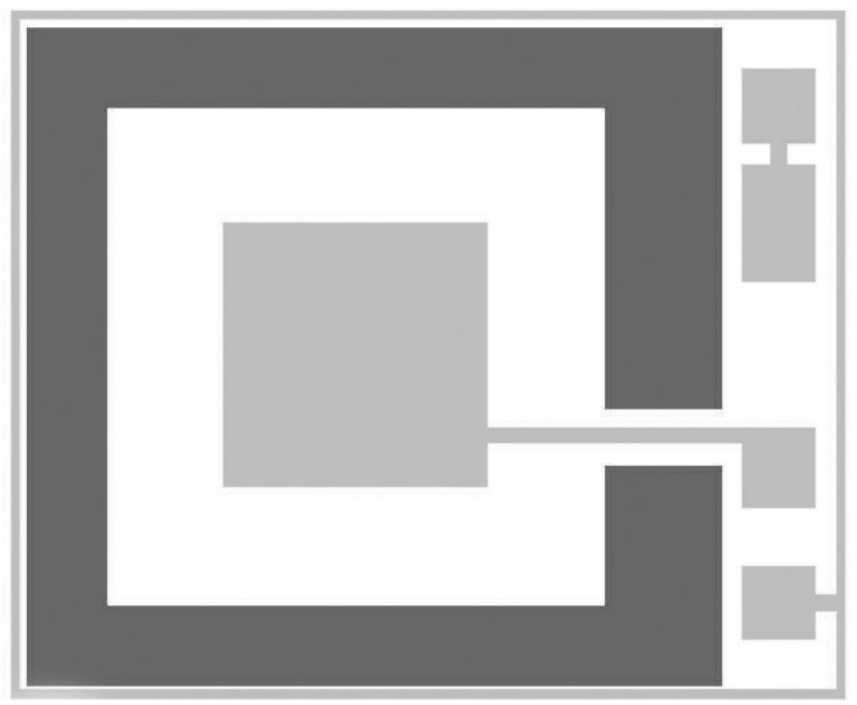

Al

Si

Fig. 5. Patterned carrier glass and the Si electrode alignment.

The fabrication of the fixed metal electrode is based on two significant processing steps. First a $1 \mu \mathrm{m}$ thick aluminum layer was deposited on Borofloat ${ }^{\circledR} 33$ glass by electron beam vacuum evaporation, and patterned by conventional aluminum etching subsequently. The pressure sensors suitable for detecting various pressure ranges can be tailored by the accurate control of the membrane's thickness and the electrode areas of the capacitor. In our case three different sensor structures were fabricated applying $1.44 \mathrm{~mm}^{2}, 3.24 \mathrm{~mm}^{2}$ and $5.76 \mathrm{~mm}^{2}$ electrode areas for 0-1000 mbar pressure ranges. Fig. 5] shows the alignment of the metal pattern on the glass substrate and the bottom side shallow cavity of the Si electrode.

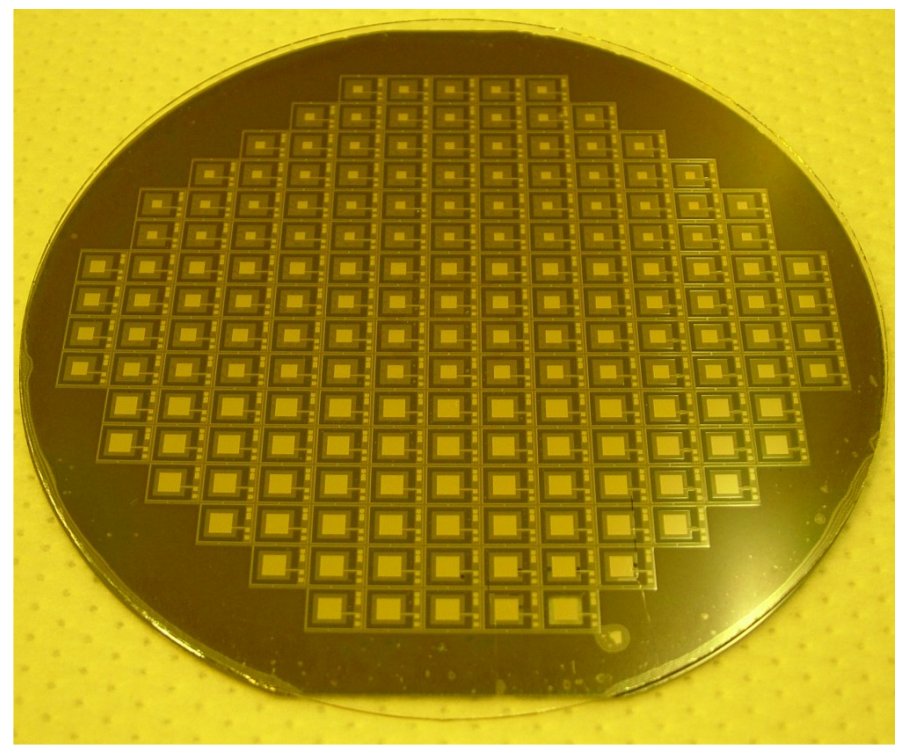

Fig. 6. 4" size structured, bonded wafer pair.

\section{Wafer alignment and anodic bonding}

The pre-bond alignment of the 4 inch silicon and glass wafers was carried out by using a Mask Aligners/Bond Aligners MA6/BA6 type equipment made by SÜSS MicroTec AG (Germany). The wafers were bonded anodically [7, 8] in a SÜSS SB6L wafer bonder machine. The parameter approaches of the achieved experiments applicable for reproducible bonding the aligned 4" wafers are summarized in Table 2, and the successfully bonded wafer pair is shown in Fig. 6

Tab. 2. Anodic bond parameters for 4 " wafers.

\begin{tabular}{lcc}
\hline Temperature & $T=200^{\circ} \mathrm{C}$ & \\
Voltages & $U_{1}=-500 \mathrm{~V}$ & $U_{2}=-1000 \mathrm{~V}$ \\
Time & $t_{1}=1 \mathrm{~min}$ & $t_{2}=40 \mathrm{~min}$ \\
Vacuum & $p_{v}=10^{-3} \mathrm{mbar}$ & \\
Tool Pressure & $p_{t}=1000 \mathrm{mbar}$ & \\
\hline
\end{tabular}

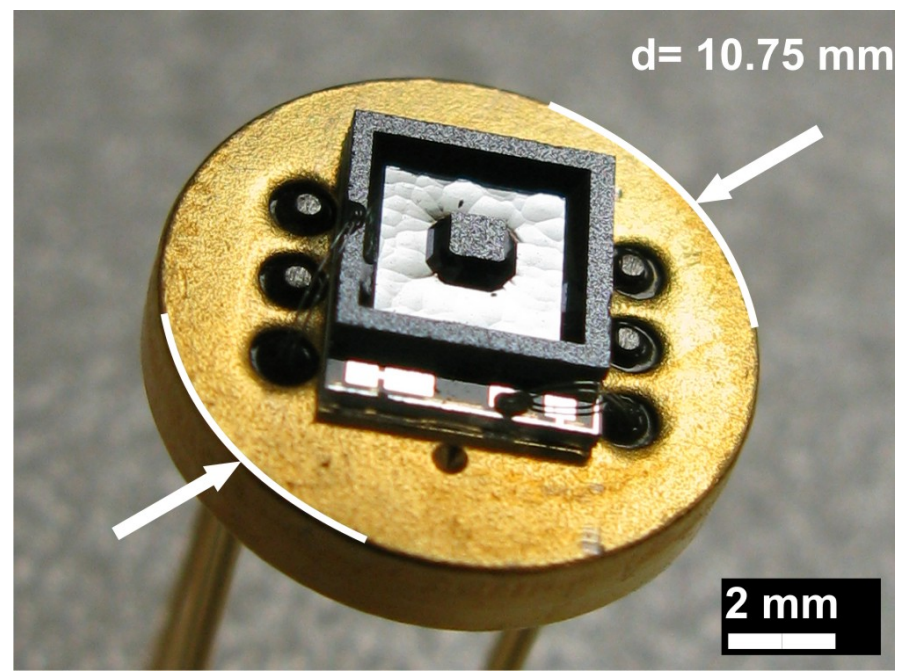

Fig. 7. The packed relative pressure sensor. 
Tab. 3. The results of the chips measurement.

\begin{tabular}{ccccc}
\hline Chip & $\begin{array}{c}\text { Si-Al } \\
\mathrm{R}[\mathrm{M} \Omega]\end{array}$ & $\begin{array}{c}\text { Repose Cap. } \\
C_{r e p}[p F]\end{array}$ & $\begin{array}{c}\text { Loaded Cap. } \\
C_{l}[p F]\end{array}$ & \\
\hline$\# 1$ & $\infty$ & 6.452 & 17.42 & \\
$\# 2$ & $\infty$ & 6.419 & 21.34 & large \\
$\# 3$ & $\infty$ & 6.416 & 19.76 & \\
$\# 4$ & $\infty$ & 6.411 & 20.80 & \\
$\# 5$ & $\infty$ & 6.376 & 20.17 & \\
\hline$\# 6$ & $\infty$ & 3.573 & 11.41 & medium \\
$\# 7$ & $\infty$ & 3.513 & 11.98 & \\
\hline$\# 8$ & $\infty$ & 2.292 & 6.41 & \\
$\# 9$ & $\infty$ & 2.258 & 5.79 & \\
$\# 10$ & $\infty$ & 2.241 & 5.56 & \\
$\# 11$ & $\infty$ & 2.230 & 6.62 & \\
$\# 12$ & $\infty$ & 2.223 & 5.88 & \\
\hline
\end{tabular}

The bonded silicon/glass structures were diced for chips and tested electrically. The selected MEMS devices were packaged onto a TO8 socket (Schott) as presented in Fig. 7. The wire connects between bonding pads of the sensor and the socket were realized by ultrasonic-compression bonding applying $50 \mu \mathrm{m} \mathrm{di}$ ameter Al wires. In the last processing step, the shallow silicon trench serving as buried wiring channel for the metal electrode was filled up by non-permeable glue to form the separated pressure chamber. Relative pressure sensor was formed by hermetic sealing of the cavity from the outer ambient.

\section{Results and discussion}

\subsection{Strength of the anodic bonding}

The bonded area in a chip is $9.503 \mathrm{~mm}^{2}$; the bond strength between the two electrodes is $25 \pm 5 \mathrm{MPa}$. The result was assessed with a tensile strength measurement method, carried out on 10 distinct samples. Fig. 8 shows the surfaces of a sample after the tensile measurement.

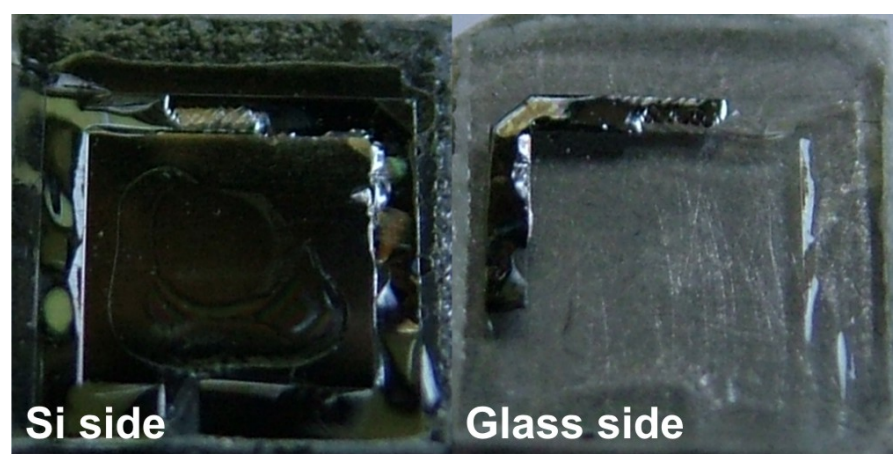

Fig. 8. Bonded surface after tensile strength measurement: the silicon surface (left), the glass surface (right).

\subsection{Results of chips measurement}

Before the packaging the zero-pressure capacitances of the samples were measured with HP Frequency RLC Meter type 4275A using a Karl SÜSS type pin tester (Fig. 9). Table 3. shows the results of the measurement. One can observe that after wafer dicing the chips are functional, i.e. the measured capacitance responds to the pressure change and at zero pressure it returns back to the initial value.

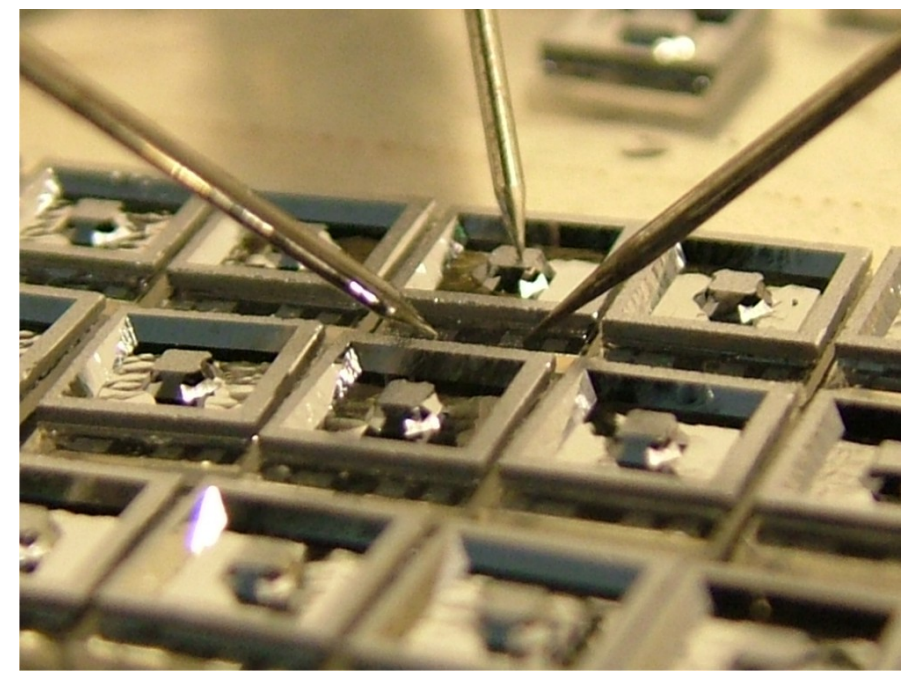

Fig. 9. The pre-shorting measurement with the pin tester before packaging.

\subsection{Functional measurements of packed chips}

The realized devices were functionally tested in the pressure range of 0 to 1000 mbar. Fig. 10 shows the capacitance calibration curves of the different sensors as the function of the pressure load from 0 to 500 mbar. The sensitivity of the pressure sensor was calculated from the measured results which is $6 \mathrm{fF} / \mathrm{mbar}$. The linearity of capacitance change turns to nonlinearity approximately above 400 mbar in case of "small" and "medium" electrode sizes. It means these structures are not reliable at pressures higher than 500 mbar. However, the chip with the "large" electrode size works linearly up to 800 mbar.

\section{Conclusion}

The feasibility of a capacitive type MEMS pressure sensor was demonstrated. Proper silicon membrane homogeneity and thickness variation was achieved by the application of the combined alkaline etching and Electrochemical Etch-Stop techniques. The low-cost silicon/glass wafer bonding process ensured reliable mechanical stability and hermetic sealing. We have compared the results of simulations to the measured values, and we have found a good correlation between the electrode size and the working pressure range. Nevertheless, thinner membranes (e.g. $10 \mu \mathrm{m}$ ), with same electrode size and center mass reinforcements should be realized and tested in the range of 0-1000 mbar. 
Fig. 10. Calibration curves of the different capacitive pressure sensors.

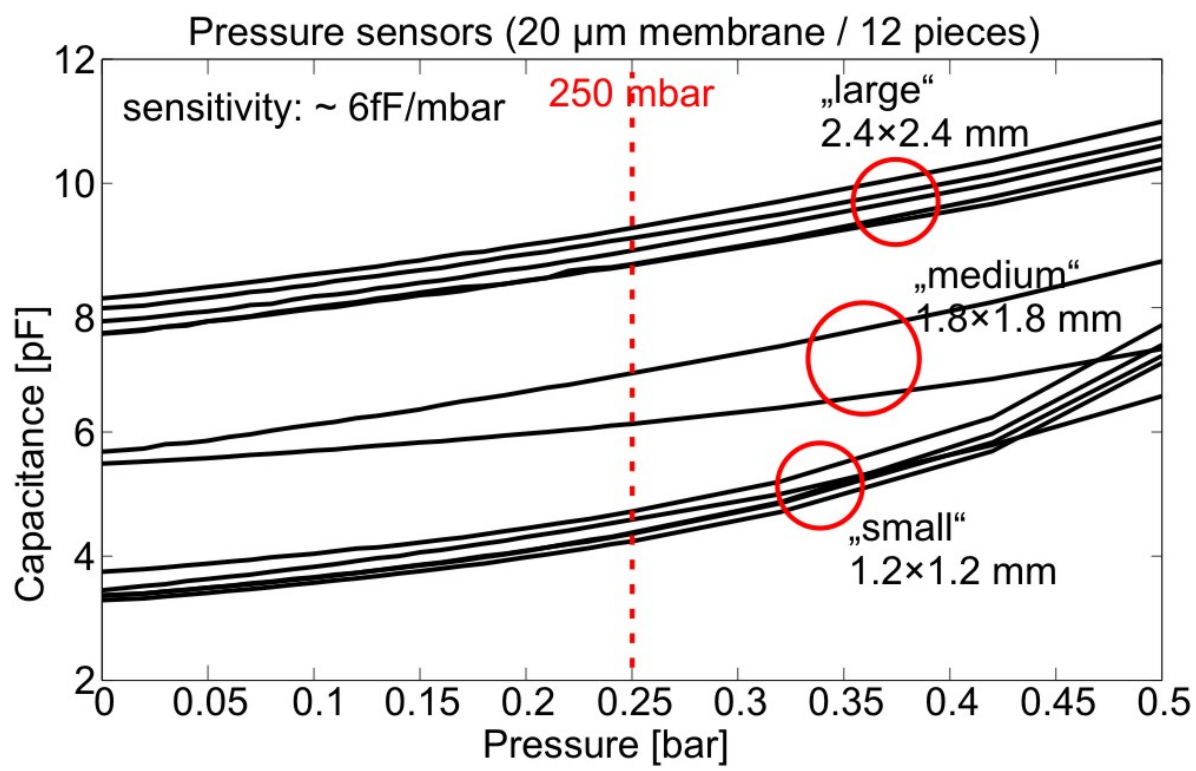

\section{References}

1 Liao S, Chen W, Lu M, A CMOS MEMS Capacitive Flow Sensor for Respiratory Monitoring, IEEE Sensors Journal, 13(5), (2013), 1401-1402, DOI 10.1109/JSEN.2013.2245320.

2 Eaton W, Smith J, Micromachined pressure sensors: review and recent developments, Smart Mater. Struct., 6, (1997), 530-539.

3 Narducci M, Yu-Chia L, Fang W, Tsai J, CMOS MEMS capacitive $a b$ solute pressure sensor, Journal of Micromechanics and Microengineering, 23(5), (2013), 055007-055007, DOI 10.1088/0960-1317/23/5/055007.

4 Gopel W, Hesse J, Zemel JN, Sensors, A Comprehensive Survey, Volume 7., VHC Publishers Inc., USA, 1993.

5 Performance proceeding between $M M G-A M$ and MTA ATKI, performance report, MTA-MFA; Budapest, 1997-11-30.

6 Wallman L, Bengtsson J, Danielsen N, Laurell T, Electrochemical etchstop technique for silicon membranes with $p$ - and n-type regions and its application to neural sieve electrodes, J. Micromech. Microeng., 12, (2002), 265-270, DOI 10.1088/0960-1317/12/3/311.

7 Wallis G, Pomerantz DI, Field assisted glass-metal sealing, J. Appl. Phys., 40(11), (1969), 3946-3949.

8 Li GY, Wang L, Influence of bonding parameters on electrostatic force in anodic wafer bonding, Thin Solid Films, 462, (2004), 334-338, DOI 10.1016/j.tsf.2004.05.084. 